\title{
Cooperative sensors: a new wired body-sensor-network approach for wearable biopotential measurement
}

\author{
Michael Rapin \\ CSEM \\ Jaquet-Droz 1 \\ CH-2002 Neuchâtel \\ +41327205420 \\ michael.rapin@csem.ch
}

\author{
Josias Wacker \\ CSEM \\ Jaquet-Droz 1 \\ CH-2002 Neuchâtel \\ +41327205663 \\ josias.wacker@csem.ch
}

\author{
Olivier Chételat \\ CSEM \\ Jaquet-Droz 1 \\ CH-2002 Neuchâtel \\ +4132 7205313 \\ olivier.chetelat@csem.ch
}

\begin{abstract}
Cooperative sensors are a novel measurement architecture based on active dry electrodes that allows the acquisition of biopotential signals (e.g., electrocardiogram, ECG) on patients in a comfortable and easy-to-integrate manner. This paper starts by analyzing the classical methods for measuring multilead ECG signals. Based on these classical methods, we then briefly introduce the concept of cooperative sensors and show how these sensors can be implemented in a wearable and reliable system that measures ECG signals in a real-life scenario. The main focus and innovation presented in this paper is on the duplex communication between the cooperative sensors. In addition, we show a first measurement of an ECG signal with cooperative sensors.
\end{abstract}

\section{Keywords}

Wearable sensors, active electrodes, dry electrodes, cooperative sensors, body sensor network (BSN), electrocardiogram (ECG), multilead.

\section{INTRODUCTION}

In recent years, many different sensor systems for measuring physiological signals have been developed and placed on the market. First sensor designs were relatively bulky and mainly used in bed-side monitoring units. Today, however, there is an increasing demand for systems which have similar or better specifications than the best bed-side systems (e.g., number of measured signals, high signal quality) and can be worn in daily life [1]. One of the main challenges in the development of wearable monitoring systems is the size and weight reduction of its elements (sensors, centralized box with control and recording electronics, cabling, connectors, supporting structure). Additionally, the integration (in particular the cabling) of the sensors in a wearable monitoring device should be simple from a manufacturing and usage point of view.

In this paper, we introduce cooperative sensors, a novel sensor architecture which allows overcoming the current limits in integration [2]. We start by presenting the classical methods for multilead ECG measurement. Then, a link is made from the classical architecture to cooperative sensors. Finally, the duplex communication between cooperative sensors is presented.

\section{Classical methods}

Figure 1 shows the classical architecture for measuring multilead ECG signals. In this configuration passive electrodes are linked to a centralized electronics box via shielded cables. The potential of the shield follows the potential of the cable core to keep the input impedance of each ECG lead as high as possible [3, 4]. A guard electrode and the common mode controller $H$ are used to reduce the common-mode voltage that is usually induced by external electromagnetic perturbations.

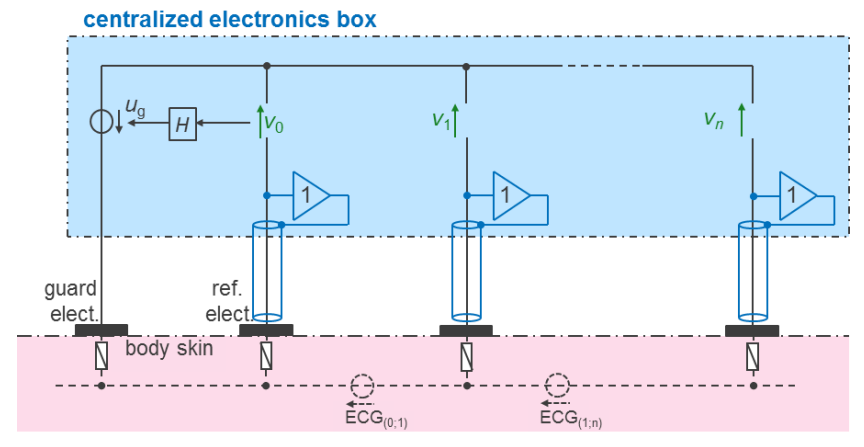

Figure 1. Classical architecture for measuring multilead ECG signals, comprising a centralized electronics box

A first alternative to shielded cables uses active electrodes. In this configuration, the analog front end (AFE) is moved close to the skin electrode location [5]. This way, the length of the cable sensitive to electromagnetic noise is drastically reduced. Nevertheless, this architecture requires multi-wire cables to link active electrodes to a centralized electronics box.

Both, passive and active electrode architectures require the use of a centralized electronics box to which all cables converge. As a consequence, for a large number of leads, the miniaturization of the centralized electronics box is limited by the size of cables and connectors, and the production of multi-sensor systems is relatively complex.

\section{COOPERATIVE SENSORS}

\subsection{Architecture}

In order to overcome the limitations of the architectures which include a centralized electronics box, a novel design, so-called 
cooperative sensors, has been presented in [2]. Cooperative sensors use the same underlying electrical circuit as the classical architecture presented in Figure 1. However, as shown in Figure 2 , the centralized electronics box has been replaced by two types of active cooperative sensors: 1) one reference sensor, which has two contacts with the skin and also plays the role of guard electrode [2], and 2) an arbitrary number of measuring sensors, which have only one contact with the skin. In this configuration each sensor has its own power supply (e.g., a battery) and active electronic components.

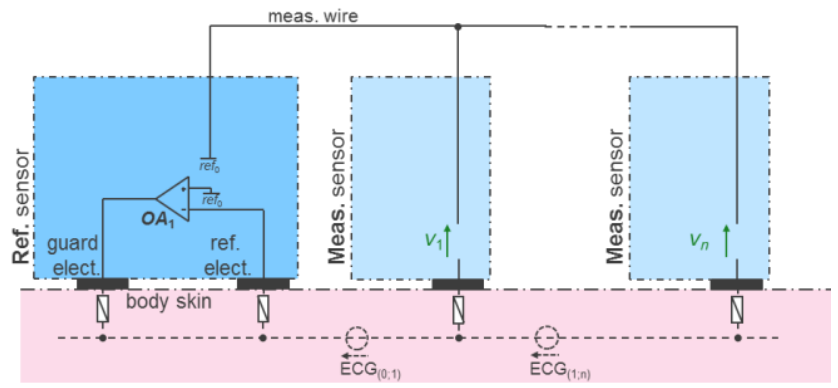

Figure 2. Cooperative-sensor architecture for measuring multilead ECG signals without centralized electronics box

In Figure 2, all cooperative sensors are linked by the same external connection wire, the measurement wire (or meas. wire). The electrical potential of this wire is set to the body potential picked-up under the reference electrode of the reference sensor thanks to the operational amplifier $O A_{1}$ [2]. Each measuring sensor measures the voltage difference between its skin electrode location and the measurement wire (i.e., the body potential at the reference electrode location). Thus, analog signal processing (i.e., amplification and filtering) of the potentials $v_{1} \ldots v_{n}$ and analog-todigital conversions are performed on-site in each measuring sensor.

\subsection{Communication principle}

In the following section, the two directions of communication between the cooperative sensors are presented: from the reference sensor to the measuring sensors (for synchronizing the sensors) and from the measuring sensors to the reference sensor (for concentration of data).

\subsubsection{Synchronization of the sensors}

Since each measuring sensor acquires and digitizes an ECG signal $\left(v_{1} \ldots v_{n}\right)$ at its specific location, all cooperative sensors need to share the same time basis [6]. To that end, a second wire, the communication wire (comm. wire) is added (see Figure 3). Through this communication wire, the synchronization signal $u_{0}$ is sent to all measuring sensors $\left(u_{1} \ldots u_{n}\right)$.

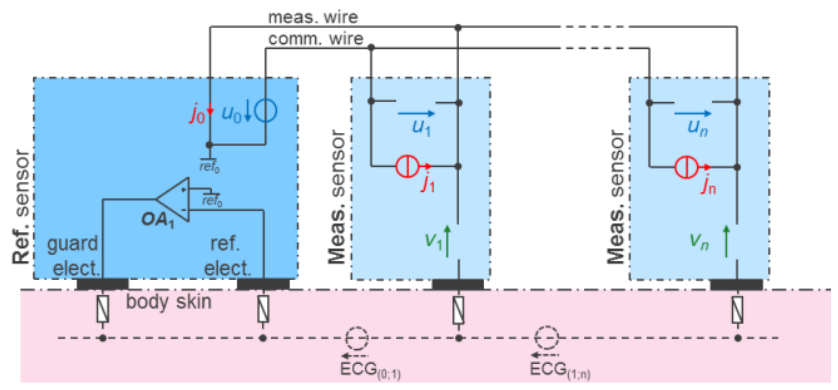

Figure 3. Communication principle of cooperative sensors
As shown in Figure 4, the synchronization signal is sent as small voltage impulses generated by the voltage source $u_{0}$ of the reference sensor. By setting two threshold voltages $u_{+}$and $u_{-}$(also shown in Figure 4) in each measuring sensor, the received signal $\left(u_{1} \ldots u_{n}\right)$ can easily be digitized to get the signal $u_{\#}$. To that end, when the $u_{+}$threshold is reached, the $u_{\#}$ signal is set and when the $u_{-}$threshold is reached, $u_{\#}$ is reset. This digitized communication signal is then used by each measuring sensor to recover the clock (frequency and phase) of the reference sensor thanks to a PLL.

It is worth noting that the signal $u_{0}$ could also be used by the reference sensor to transmit information to the measuring sensors, for instance to adjust the gain or other measurement parameters of measuring sensors.
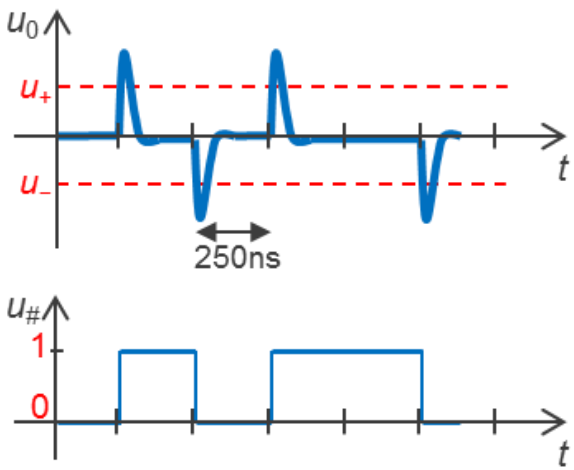

Figure 4. Synchronization and communication signals $u_{0}$ and $u_{\#}$

As shown in Figure 5, the synchronization frequency band is well separated from the frequency band of the measured ECG signal.

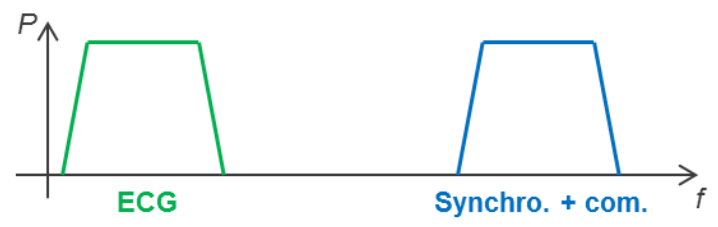

Figure 5. Frequency band allocation for ECG and synchronization/communication signals

\subsubsection{Data concentration}

Since the ECG signals $v_{1} \ldots v_{n}$ are locally digitized in each measuring sensor, means of communication between the measuring sensors and the reference sensor are required to allow concentration of data. This communication is performed with the current sources $j_{1} \ldots j_{n}$ (see Figure 3 ). The information is sent as current impulses with similar shape as the voltage signal $u_{0}$ and in the same frequency band. This current signal is sensed in the reference sensor as $j_{0}$ and a similar threshold method as for the voltage signal is used to digitize the current signal. Since all current sources work in parallel, each measuring sensor transmits its digitized measured signal at a pre-defined time to avoid current overlap.

\subsubsection{Comparison to the state-of-the-art communication in body sensor networks}

In opposition to other wired or semi-wired body sensor networks reviewed in the literature $[7,8,9]$, the cooperative-sensor architecture allows simultaneous bidirectional communication between cooperative sensors with only two unshielded wires. Furthermore, one of these two wires (the meas. wire) is also used 
as a voltage reference to sense the measured biopotential (i.e., ECG signal).

It is worth noting that each cooperative sensor has only two contacts with the external connection wires. As a consequence, the size of the sensors is not determined by the number of leads.

\section{PRELIMINARY RESULTS}

The communication principle presented in section 3.2 has been implemented and tested with electronic breadboards. Figure 6 shows the synchronization signal $u_{0}$ sent by the reference sensor and measured between the two external wires by all measuring sensors. The voltage peak amplitude is approximately $\pm 60 \mathrm{mV}$ and the threshold voltages have been set to $\pm 30 \mathrm{mV}$.

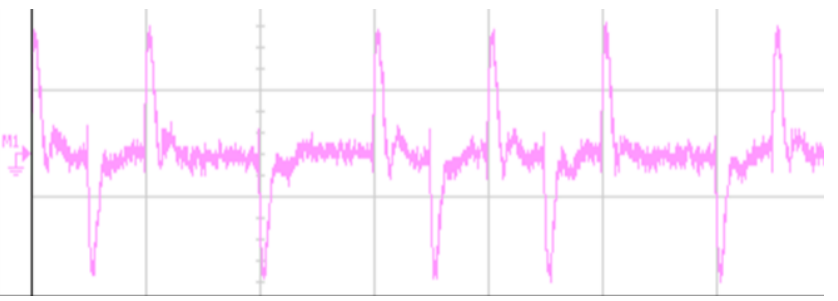

Figure 6. Synchronization signal $u_{0}(50 \mathrm{mV} / \mathrm{div}$.; 500ns/div.)

As shown in Figure 7, the synchronization signal $u_{0}$ is generated by a digital signal $u_{\mathrm{Tx}}$ filtered by a first order band-pass filter with a central cutoff frequency of $5 \mathrm{MHz}$. This band-pass filter is implemented with the operational amplifier $\mathrm{OA}_{2}$.

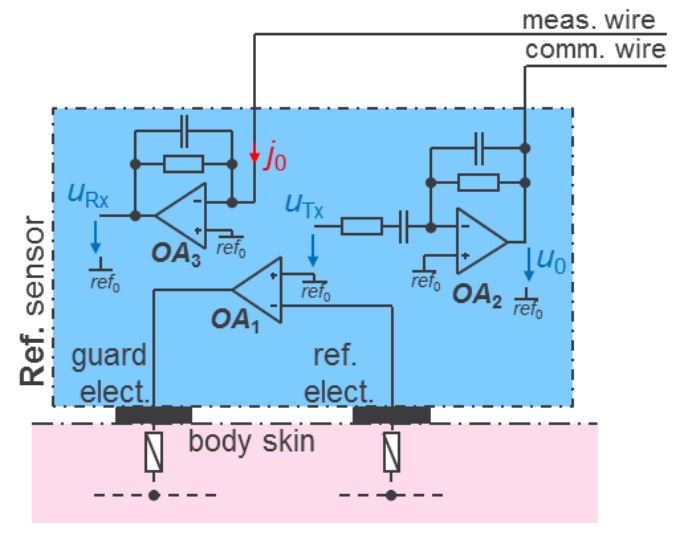

Figure 7. Detailed electronics of the reference sensor

Figure 8 shows the digital signal sent by the reference sensor $\left(u_{\mathrm{Tx}}\right)$ and the signal $u_{\#}$ recovered by one of the measuring sensor after the threshold comparators. The delay time induced by the line drivers and the underlying comparators is around $75 \mathrm{~ns}$.

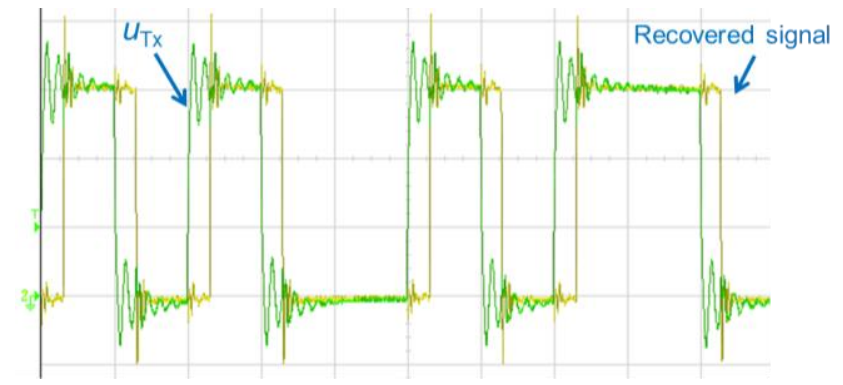

Figure 8. Sent $\left(u_{\mathrm{Tx}}\right)$ and recovered $\left(u_{\#}\right)$ communication signal (1V/div.; 250ns/div.)
A first actual measurement with cooperative sensors has also been performed. The ECG signal was generated by an ECG simulator (SecuLife PS200) and the measured signal is shown in Figure 9. This one-lead ECG signal proves that the communication principle described in this paper works well and does not interfere with the ECG signal, and that the unshielded wires that link sensors together are not sensitive to external electromagnetic noise (i.e., $50 \mathrm{~Hz}$ mains perturbations). The digital signal resolution is $200 \mathrm{nV} / \mathrm{LSB}$ and the bandwidth goes from $0.05 \mathrm{~Hz}$ to $150 \mathrm{~Hz}\left(1^{\text {st }}\right.$ order filters).

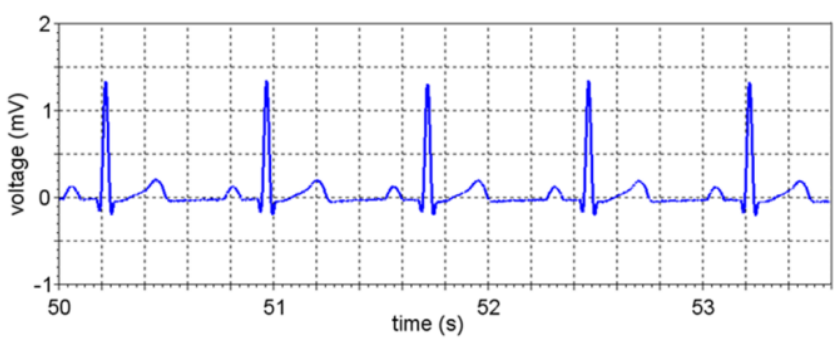

Figure 9. One-lead ECG signal measured with cooperative sensors on an ECG simulator

\section{FUTURE IMPLPEMENTATION}

\subsection{Reference sensor}

Figure 10 shows the final implementation of the reference sensor. This sensor has two contacts with the skin which are implemented as a central pad (for the reference electrode) and an external ring (for the guard electrode). The communication and the measurement wire are connected to the sensor via snap buttons placed on the back of the sensor.

In the designed implementation, the reference sensor has additional sensing functionalities, namely an accelerometer for measuring body movements of the wearer and LED/photodiode system for quantifying the blood oxygen saturation (SpO2) [10].

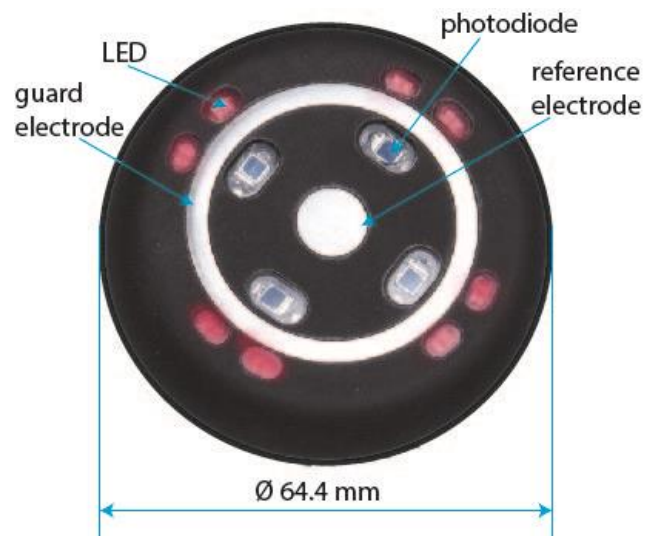

Figure 10. Implementation of the reference sensor with two electrodes and LEDs/photodiodes for $\mathrm{SpO} 2$ measurement

\subsection{Measuring sensors}

In contrast to the reference sensor, measuring sensors only have one contact with the skin. The connection with the communication and the measurement wires is also done with snap buttons. Figure 11 shows a drawing of the implementation of the measuring sensors. 


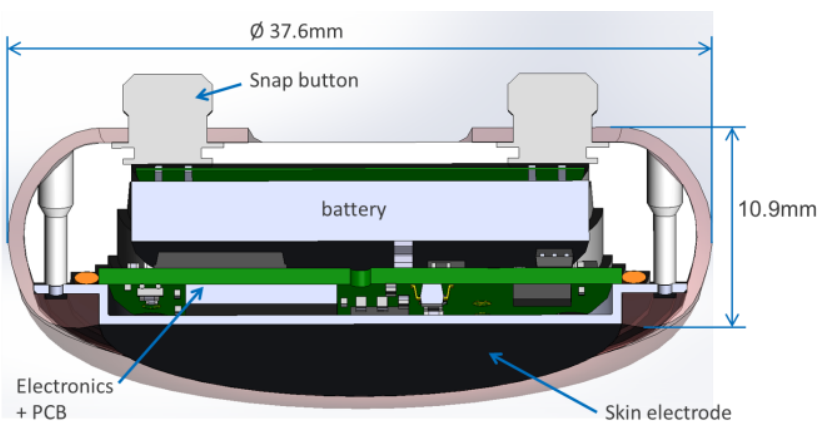

Figure 11. Final implementation of a measuring sensor

\subsection{Final system implementation}

The cooperative sensor architecture will be applied to integrated biopotential and bioimpedance measurement systems, among others. Figure 12 shows the implementation of 50 mockups of cooperative sensors in a vest [10]. Besides providing a multilead ECG, SpO2, chest sound and activity data, the entirely wearable systems will allow recording electro-impedance tomography (EIT) images of the thorax with unprecedented resolution. In this configuration the sensors are attached to the garment using their two snap buttons, and the communication and measurement wires are simply conductive wires integrated in the textile of the garment.

Both reference and measuring sensors are currently in manufacturing process and first results for this fully integrated measurement system are expected for the end of 2015.

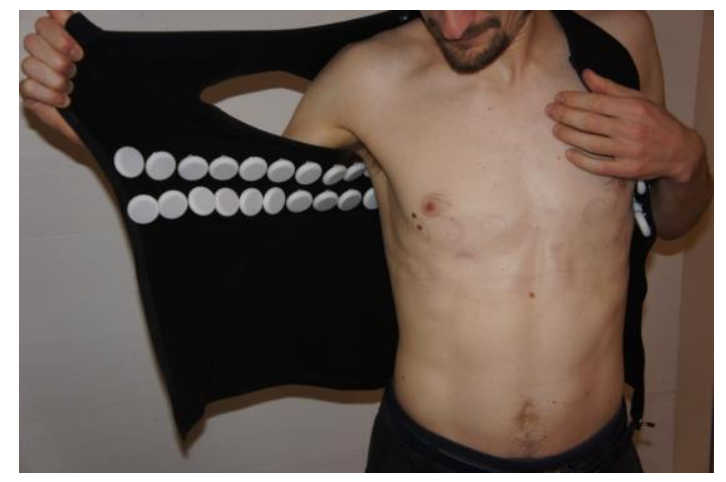

Figure 12. Final implementation of 50 cooperative sensors in a vest for measuring multilead ECG and EIT

\section{CONCLUSION}

In conclusion, we have presented a novel sensor architecture, which allows further reducing the size of the sensors and the manufacturing effort of the sensor system. The main advantage of these cooperative sensors is the simple cabling between them (done with only one non-shielded 2-wire bus). We have described in detail the frontends of the two-way communication between the sensors and showed first results. The system can now be extended to 12 leads and tested in real-life scenario. While this article focused on the application of cooperative sensors in ECG measurement, the same architecture is fully applicable to the assessment of other physiological signals (e.g., EEG, EIT).

\section{ACKNOWLEDGMENTS}

The authors would like to thank the European Space Agency (grant 4000109393/13/NL/PA) and the European commission (FP7-ICT-2013-10 project WELCOME) for the funding of this research.

\section{REFERENCES}

[1] Zheng, Y. L., Ding, X. R., Yan Poon, C. C., Lai Lo, B. P., Zhang, H., Zhou, X. L., Yang, G. Z., Zhao, N., Zhang, Y. T., 2014. Unobtrusive sensing and wearable devices for health informatics. IEEE trans. on biomed. eng. Vol. 61 (5), 153854.

[2] Rapin, M., Proença, M., Braun, F., Meier, C., Solà, J., Ferrario, D., Grossenbacher, O., Porchet, J.-A. and Chételat, O., 2015. Cooperative dry-electrode sensors for multi-lead biopotential and bioimpedance monitoring. IOP Publishing, Physiol. Meas. Vol. 36 (4), 767-83.

[3] Zipp, P., Ahrebs, H., 1979. A model of bioelectrode motion artefact and reduction of artefact by amplifier input stage design. J. Biomed. Eng. Vol. 1 (4), 273-6.

[4] Degen, T., Jackel, H., 2008. Continuous monitoring of electrode-skin impedance mismatch during bioelectric recording. IEEE Trans. Biomed. Eng. Vol. 55 (6), 1711-5.

[5] MettingVanRijn, A. C., Kuiper, A. P., Dankers, T. E., Grimbergen, C. A. 1996. Low-cost Active Electrode Improves the Resolution in Biopotential Recordings 18th Annual Int. Conf. of the IEEE Eng. in Med. and Biol. Soc., Amsterdam (NL).

[6] Proença, M., Rapin, M., Braun, F., Solà, J., Lemay, M., Thiran, J.-P., 2014. Cardiac Output Measured by Electrical Impedance Tomography: Applications and Limitations, IEEE BIOCAS 2014, Lausanne (CH), October 2014.

[7] Seyedi, M., Kilbert, B., Lai, D. T. H., Faulkner, M. 2013. A survey on intrabody communications for body area network applications. IEEE Trans. Biomed. Eng. Vol. 60 (8), 206779.

[8] Chételat, O., Rapin, M., Meier, C., Bischof, A., Augustyniak, M. K. 2015. Synchronization and communication of cooperative sensors. 37th Annual Int. Conf. of the IEEE Eng. in Med. and Biol. Soc., Milano (It).

[9] Mazioum, N. S., 2008. Body-coupled communication: experimental characterization, channel modeling and physical layer design. M.S. thesis, Dept. Sign. \& Syst., Chalmers Univ., Gothenburg, Sweden.

[10] Wacker, J., Chételat, O., Rapin, M., Meier, C., Porchet, J.A., Chang, Y. L., Pierscionek, B. K., Kayyali, R., Elnabhani, S., Philip, N., 2014. Electrical and Mechanical Design of a Vest Measuring a Large Set of Physiological Signals. 4th Int. Conf. on Wireless Mobile Communication and HealthcareMobiHealth, Athens (GR), November 2014. 